\title{
Nucare, une intervention de formation aux habiletés d'adaptation pour les patients en oncologie et leurs proches: les motivations et les attentes des participants
}

\author{
Par Linda Edgar et Suzanne Watt
}

\section{Abrégé}

Cette étude examine les rapports entre l'intention, l'auto-efficacité et les croyances en tant qu'éléments de la capacité d'apprentissage chez les sujets faisant face à un diagnostic de cancer qui ont participé au programme Nucare, une intervention de formation aux habiletés d'adaptation. Les sujets ont répondu aux questions d'une brève enquête et/ou participé à un groupe de réflexion dans le cadre de l'intervention. Nous avons constaté des rapports positifs entre le degré d'utilité que les participants accordaient à l'intervention et leurs niveaux d'auto-efficacité et de croyance dans leur capacité à modifier leurs stratégies de coping.

Les patients croient que le coping est d'une grande importance pour leur bien-être, et la compréhension de ce fait aide les infirmières à planifier des interventions permettant d'enseigner des habiletés d'adaptation aux patients et à leur famille et d'en promouvoir l'utilisation. Le programme Nucare pourra être sélectionné à titre de guide de pratique utile.

Selon Santé Canada (1997), le cancer constitue la deuxième cause de mortalité au Canada. Ainsi, les Statistiques canadiennes sur le cancer (2003) rapportent que $41 \%$ des hommes et $38 \%$ des femmes développeront un cancer au cours de leur vie et que $27 \%$ des hommes et $23 \%$ des femmes mourront de cette maladie. Le mot "cancer" fait naître des sentiments de détresse chez les patients, les membres de la famille et les amis, depuis le moment du diagnostic jusque bien après l'achèvement du traitement. Des recherches portant sur la détresse psychologique ont permis d'observer des niveaux de détresse émotionnelle cliniquement importants chez environ un tiers des échantillons de sujets atteints de cancers variés à divers points de la trajectoire du cancer (Farber, Weinerman et Kuypers, 1984; Trask et al., 2001). Quoique le dépistage soit recommandé afin de détecter les patients à risque en matière de détresse, la concordance entre les évaluations effectuées par les patients et celles effectuées par le personnel soignant n'a guère été encourageante (Trask et al., 2002). Les patients semblent souvent avoir le don de détecter leur propre détresse (Trask et al.), et ils sont capables de rechercher les soutiens et les services d'enseignement disponibles et de les mettre à profit (Edgar, Remmer, Rosberger et Fournier, 2000). En particulier, ils font des efforts pour changer leurs circonstances actuelles ainsi que leur humeur, leur santé, leurs sentiments ou leur fonctionnement social en se tournant vers leurs stratégies d'adaptation. Très peu de travaux de recherche ont examiné les facteurs liés aux décisions qui amènent les patients à participer à des programmes psychopédagogiques et aux perceptions qu'ils ont relativement aux résultats de leur participation. Qu'est-ce qui fait qu'une personne est prête à prend part à un tel programme et qu'est-ce qui contribue à sa perception de réussite?

Cet article a pour but d'examiner la participation des patients à Nucare, un programme de formation psychopédagogique aux habiletés d'adaptation, et de décrire les éléments du programme.

\section{Contexte}

Le coping

Le coping est défini par Folkman et Lazarus (1988) comme "les efforts cognitifs et comportementaux (mis en œuvre) pour gérer des demandes externes et/ou internes spécifiques qui sont évaluées comme menaçant ou excédant les ressources de la personne" (Folkman et Lazarus). Les éléments spécifiques de ces efforts sont constamment modifiés afin d'accommoder le changement continuel de la relation personne-environnement telle qu'évaluée et réévaluée par le sujet. Il en ressort que le coping engage des stratégies de comportement, de pensée ou d'action qui vont modifier la situation entraînant la détresse. Il peut s'agir de stratégies axées sur les problèmes si la situation peut être modifiée ou de stratégies axées sur les émotions dans le cas contraire.

\section{Facteurs touchant les résultats d'apprentissage}

Devine et Westlake (1995) ont étudié les effets des interventions psychopédagogiques chez les adultes atteints de cancer en réalisant une méta-analyse de 116 études. Ils ont découvert que de nombreux types d'interventions psychopédagogiques étaient bénéfiques puisqu'ils réduisaient l'anxiété, la dépression, la nausée et la douleur tout en augmentant les connaissances des individus et en les aidant à faire face aux maladies chroniques et aux maladies à issue mortelle. Certains types correspondent à des groupes de soutien offrant des séances individuelles et/ou collectives tandis que d'autres concernent des interventions psychosociales; certains autres privilégient les interventions cognitivo-comportementales alors que d'autres encore combinent une partie ou l'ensemble des techniques ci-dessus. Une recension des écrits révèle que les différentes interventions réussissent bien. Par contre, peu de travaux ont porté sur la manière dont le processus d'apprentissage se produit et quels facteurs individuels ont une incidence sur le changement de comportement ultérieur ou sur l'intention d'effectuer ce changement.

L'apprentissage peut amener le sujet à changer de réaction comportementale à un stimulus afin de réduire la tension engendrée par ce dernier. Pour faire passer une réaction nouvelle et désirée au niveau des habitudes, elle doit être répétée à maintes reprises. Elle fait alors partie intégrante du répertoire des réactions auquel le sujet accède facilement (Babcock et Miller, 1994). La théorie sociale cognitive nous enseigne que, pour réussir dans ce domaine, le sujet doit sentir le besoin de changer, être certain qu'il pourra changer et sentir que le résultat perçu vaut bien l'effort qu'il produira pour

Linda Edgar, RN, PhD, est professeure adjointe à l'École des sciences infirmières et au Département d'oncologie de l'Université McGilll et associée de recherche au Département d'épidémiologie et au programme L'espoir c'est la vie de l'Hôpital général juif de Montréal, Québec.

Suzanne Watt, RN, BSc, est infirmière clinicienne spécialisée en gestion de la douleur, Hôpital Royal Victoria, CUSM, et étudiante à la maîtrise en sciences infirmières, École des sciences infirmières de l'Université McGill. 
changer (Anderson, Winett et Wojcik, 2000). La motivation est la force agissante dans la majorité des activités humaines, et l'auto-efficacité est la perception qu'a le sujet de pouvoir mettre à profit les leçons apprises et les méthodes utilisées dans une situation particulière pour faire face à une autre (Jackson et Aiken, 2000). L'établissement de buts réalistes et leur atteinte développent l'auto-efficacité et favorisent une meilleure utilisation des habiletés d'adaptation. Ensemble, les concepts d'auto-efficacité et de motivation constituent des prédicteurs de résultat en matière de qualité de vie (Grahn, Stigmar et Ekdahl, 2001). Bandura (1977) définit l'attente d'efficacité comme la croyance qu'a le sujet de réussir à adopter un certain comportement qui doit produire un résultat donné. La théorie d'auto-efficacité de Bandura (1997) veut que les individus évaluent eux-mêmes leur capacité à participer à des activités d'autosoins qui entraîneront des résultats pertinents. L'auto-efficacité, ou l'attente qu'a l'individu d'assimiler des compétences appropriées, influera sur la décision du sujet d'essayer ou non d'adopter des comportements d'adaptation et/ou de continuer à les adopter.

Un des éléments des ressources personnelles en matière de coping est le sentiment de contrôle sur une situation donnée (Grahn et al., 2001). Il est nécessaire de réduire l'incertitude si on veut augmenter le sentiment de contrôle. L'incertitude peut provenir d'une compréhension insuffisante d'une situation donnée, de l'absence de solutions de rechange au problème, d'un manque d'information et du doute du sujet vis-à-vis de son rôle dans la situation ou des résultats liés au choix d'intervention. Pour composer efficacement avec l'incertitude, il convient d'obtenir davantage d'information, de demander conseil, de peser le pour et le contre, d'anticiper la situation et de simuler l'événement dans sa tête afin de mieux prévoir quelle intervention convient le mieux à une situation donnée (Lipshitz et Strauss, 1997).

Les sujets qui exercent habituellement un contrôle sur leur environnement ont tendance à manifester des degrés élevés de satisfaction, de jouissance de la vie, de confiance en eux et dans autrui et à faire preuve de davantage de persévérance et d'initiative. Ils jouissent généralement d'une meilleure santé physique et psychologique que les individus qui se sentent contrôlés par des facteurs externes et une pression interne, et ils observent mieux les ordonnances médicales qu'eux (Senécal, Nouwen et White, 2000). Selon Hardy, Barkham, Shapiro, Reynolds et Rees (1995), les résultats du traitement dépendent des attentes du patient en matière de bienfaits.

La capacité d'apprentissage dépend de l'état émotionnel du sujet. Babcock et Miller (1994) renvoient à la description de McHatton des quatre stades de l'ajustement émotionnel qui mènent jusqu'à la capacité d'apprentissage en se basant sur la hiérarchie des besoins de Maslow: impact accompagné d'anxieté, de désespoir et de perte de contrôle; régression avec déni, fuite et besoin de se sentir entouré; reconnaissance, y compris doute, perte de confiance en soi et perte d'estime de soi; enfin, reconstruction caractérisée par le besoin de retrouver un sentiment de contrôle et de confiance. Dans ce contexte, l'auto-efficacité, la confiance et l'importance perçue du coping sont des variables qui se chevauchent et qui sont des préalables à l'apprentissage de nouvelles façons de faire face aux stresseurs.

\section{L'intervention Nucare}

Nucare (dont le nom vient des deux premières lettres des mots anglais "nursing", "cancer" et "research") est une intervention psychosociale et pédagogique offerte aux patients atteints de cancer et à leurs proches afin de les aider à développer les compétences utiles pour faire face à cette maladie. L'intervention se compose de trois séances de deux heures dans le cadre desquelles on aborde les sept habiletés d'adaptation suivantes: résolution active de problème, façons de penser, communication, utilisation du soutien social, établissement de buts, habitudes de vie saines et formation à la relaxation. Nous présentons ci-dessous une brève description des éléments particuliers de Nucare:
1) Techniques de résolution de problème: On enseignait à chaque patient une série particulière d'étapes pour la résolution de problème. On utilisait d'abord un exemple neutre pour illustrer les étapes et s'entraîner avant d'utiliser les problèmes des patients dans le cadre de l'application pratique des techniques.

2) Établissement de buts: L'établissement de buts progressifs et atteignables constitue un moyen réaliste d'accomplir des tâches qui sont opportunes et pertinentes et grâce auxquelles le sujet éprouve un sentiment de contrôle personnel. On encourageait les patients à se fixer des buts à court ou à moyen terme.

3) Réévaluation cognitive: On apprenait aux patients à prendre davantage conscience de leurs modes de pensée et de repérer ceux qui engendrent une humeur négative. Pour les patients, le seul fait d'apprendre qu'ils peuvent contrôler leurs pensées était souvent tout ce dont ils avaient besoin pour amorcer un changement positif et améliorer leur humeur. On invitait les patients à s'entraîner à distinguer les faits des pensées et des sentiments. Tout d'abord, les patients reconnaissaient que leurs sentiments désagréables ne provenaient pas seulement des faits mais aussi de l'évaluation cognitive qu'ils faisaient eux-mêmes de leur situation. Ensuite, on a enseigné aux patients comment réévaluer ou recadrer leurs pensées négatives, déformées ou catastrophiques afin qu'il leur soit plus facile de les contrôler et qu'elles engendrent une détresse moindre. On soulignait les rapports entre la réévaluation cognitive et un sentiment accru de contrôle personnel.

4) Formation à la relaxation: Il a été clairement démontré que la relaxation est une stratégie de traitement efficace pour les conditions liées au stress. La formation à la relaxation constituait la deuxième étape de la démarche de résolution de problème (c.-à-d. prendre le temps de concevoir une perspective différente). On utilisait le plus fréquemment la relaxation musculaire progressive associée à l'imagerie mentale dirigée. On fournissait aux patients une cassette audio pour la pratique à domicile.

5) L'utilisation efficace $d u$ soutien social: on aidait les patients à repérer des sources d'information et de soutien social y compris des sources d'aide pratique et d'aide affective et à déterminer le caractère adéquat de leur propre réseau de soutien social.

6) Communication: On a appris aux participants à utiliser des énoncés à la première personne du singulier, à développer des comportements assertifs, à dire "non", ainsi que des stratégies visant à favoriser une bonne relation médecin-patient.

7) Éléments d'un mode de vie sain: On a discuté brièvement des sujets suivants parce qu'ils ont tous une incidence sur la santé: l'espoir, la spiritualité, le rire, le régime alimentaire, l'exercice et la gestion de la fatigue.

La figure 1 présente une page tirée du cahier sur les habiletés d'adaptation utilisé dans le cadre du programme Nucare.

Le processus de la modification du comportement est abordé dans le contexte des sept compétences dégagées dans Nucare, c'est-à-dire: a) la nécessité de pratiquer de nouvelles compétences de 30 à 60 fois; b) l'impact qu'une petite dose de changement peut avoir sur le bienêtre et l'adaptation. Avant tout, Nucare permet au sujet de découvrir et d'utiliser ses propres points forts pour atteindre des buts réalistes qu'il juge importants en lui redonnant un sentiment de contrôle personnel. Les séances interactives et néanmoins didactiques permettent aux participants de poser des questions, de discuter des différentes interventions et de se soutenir les uns les autres. Chaque participant reçoit un cahier complet contenant des instructions simples, des exercices se rapportant à des situations du monde réel et à des relations interpersonnelles ainsi que des mots d'encouragement pour que les participants persévèrent dans la pratique des compétences. Les résultats d'un programme global de recherche sur Nucare réalisé dans le cadre de trois grands essais cliniques randomisés ont clairement démontré son efficacité au niveau de la promotion d'une adaptation saine et de la réduction de la détresse (Edgar, Rosberger et Collet, 2001; Edgar, Rosberger, Collet et Fournier, 2002; Edgar, Rosberger et Nowlis, 1992). 
Nucare est basé sur des données probantes issues de la recherche selon lesquelles le coping, faisant appel à la résolution de problème axée sur les buts planifiés, à la réévaluation positive et à l'adaptation combative, exerce une forte influence sur la réaction émotionnelle aux stimulus stressants, et "la résolution de problème et la réévaluation positive amélioraient l'état émotionnel." (Folkman et Lazarus, 1988, p. 314).

Le manuel du programme Nucare est un cahier d'exercices interactif de 72 pages très convivial qui guide les individus dans leur évaluation de leurs compétences actuelles et les aide à cerner les nouvelles compétences qui pourraient mieux convenir à leurs besoins. Le cahier comprend des directives pratiques, des feuilles de travail, des espaces pour la réflexion et une brève interrogation écrite sur chaque compétence. Il est d'un style simple et clair, comme on le voit à la figure 1 , et il souligne le besoin de pratiquer les nouvelles compétences (de 30 à 60 fois) ainsi que le fait qu'en matière de coping, même un petit changement donne des résultats. Des études ont montré que les interventions qui comprenaient du matériel imprimé que les patients pouvaient conserver pour consultation future avaient une plus grande incidence sur les connaissances que celles qui n'en incluaient pas (Devine et Westlake, 1995).

L'intervention Nucare est fondée sur les principes de la modification de la relation entre le sujet et son environnement à l'aide du modèle de soins infirmiers de McGill. Le coping, la capacité à faire face à une situation pénible, et la croissance humaine constituent les principales dimensions de ce construit à facettes multiples de la santé au sein du modèle. Le but du coping est de réduire la tension ou d'atténuer le problème et, en plus, d'acquérir une expertise (maîtrise) en résolution de problème. Les résultats du coping adéquat sont, entre autres, les suivants: meilleure santé, amélioration des compétences familiales en résolution de problème, meilleure satisfaction vis-à-vis des résultats des décisions prises et enfin, amélioration de la qualité de vie (Gottlieb et Rowat, 1987).

Dans le modèle de soins infirmiers de McGill, la croissance se produit chez le sujet lorsqu'il découvre les ressources actives et latentes en lui-même et dans sa famille ainsi que dans le système social environnant, active ces ressources et les maintient ou les adapte au gré des circonstances. Le sujet est un participant actif, capable d'apprendre de nouvelles manières de faire face aux situations difficiles et de résoudre des problèmes, qui comprend que les solutions se trouvent en lui et chez les membres de la famille. Ce modèle présuppose que le sujet veut assumer la responsabilité de sa propre santé et que les ressources, les forces et l'énergie nécessaires existent bel et bien chez le sujet et ses proches (Gottlieb et Rowat, 1987).

Dans ce modèle, le rôle de l'infirmière consiste à promouvoir la santé en dispensant un enseignement au moyen d'approches qui répondent aux besoins d'apprentissage du patient, à ses besoins et à son style d'adaptation. L'évaluation de l'infirmière est essentiellement validée par le patient lui-même.

\section{Méthodologie}

\section{Les participants}

Cette étude descriptive faisait appel à un échantillon de convenance de participants à l'atelier Nucare. Vingt individus ont participé à un suivi téléphonique post-intervention et/ou ont assisté à un groupe de réflexion. Ils ont assisté à deux séances collectives de Nucare de deux heures chacune et ont reçu un appel téléphonique de deux à quatre mois plus tard afin de participer à une enquête de suivi. À ce moment-là, ces participants ont également été invités à participer à un groupe de réflexion ayant lieu un mois plus tard.

Les participants pouvaient participer à l'intervention Nucare dans un des hôpitaux d'enseignement de l'Université McGill à Montréal par l'intermédiaire du département L'espoir c'est la vie, un organisme bénévole de soutien par les pairs responsable des programmes de soutien et d'enseignement destinés aux patients en oncologie et à leurs proches. Les participants avaient répondu à l'invitation lancée par voie d'affiches ou avaient été encouragés par des membres de l'équipe soignante. Cependant, la décision de participer et le choix de la date de leur participation leur revenaient à eux seuls, et ils savaient que nous nous intéressions à l'utilité qu'ils accordaient au programme et que nous solliciterions leur avis par le biais d'une enquête téléphonique.

Les bénévoles de L'espoir c'est la vie reçoivent une orientation et une formation de 20 heures pour être capables de remplir leurs rôles auprès des patients atteints de cancer et de leurs proches. Cette formation comprend la conduite de conversations téléphoniques visant à évaluer divers services offerts. Les bénévoles de L'espoir c'est la vie ont téléphoné à 27 participants à l'atelier Nucare en vue de leur administrer l'enquête de suivi et ont pu joindre 20 d'entre eux.

L'enquête conçue pour évaluer le programme se basait sur une enquête utilisée dans l'évaluation de points semblables dans une autre étude (Schwarzer et Renner, 2000). Elle comprenait six questions permettant d'évaluer les éléments suivants: la valeur de l'intervention, grâce à la question "Quel était le degré d'utilité des

\section{Figure 1: Page tirée du manuel Nucare \\ Traduction libre du texte anglais reproduit avec la permission du $D^{r}$ Edgar et de ses collaborateurs. \\ La plus grande découverte de ma génération est que les êtres humains peuvent modifier leur vie en modifiant leur état d'esprit.}

- William James

Quelle sorte d'état d'esprit?

Pas nécessairement positif, mais nécessairement non négatif

\section{Les moyens de coping liés aux émotions et ceux liés aux pensées}

Il est généralement accepté qu'émotionnellement, nous fonctionnons de la façon suivante:

\section{FAIT}

\section{$\Downarrow$}

PENSÉE

$\Downarrow$

\section{SENTIMENT}

La majeure partie de la vie se compose de faits sur lesquels nous exerçons un minimum ou pas du tout de contrôle. En effet, nous n'exerçons aucun contrôle sur des faits tels qu'être né avec des cheveux d'une certaine couleur, être victime d'un accident, se faire muter dans une autre ville pour son travail et être atteint de certaines maladies comme le cancer. Tout fait engendre en nous une pensée, de manière spontanée sans aucun effort apparent de notre part. Cette pensée donne naissance à un sentiment.

Clarifions ce concept au moyen d'un exemple.

FAIT: Une amie vous croise dans la rue et ne vous adresse pas la parole.

PENSÉE: Elle est fâchée après moi pour une raison ou pour une autre.

SENTIMENT: Colère ou frustration.

Remarquez que le sentiment provenait directement de la pensé et non du fait. Nous exerçons peu ou pas du tout de contrôle sur la plupart des faits dont notre vie est remplie, mais nous exerçons un contrôle total sur nos pensées. Plus vous sentez que vous exercez le contrôle sur une situation donnée, moins vous éprouverez de stress et d'anxiété. Vous pouvez toujours avoir une influence et un certain degré de choix. 
ateliers?"; l'intention du sujet de changer, ou l'intérêt qu'il a de modifier son coping grâce à la question "Dans quelle mesure voulezvous modifier votre mode de coping?"; sa perception de son autoefficacité grâce à la question "Dans quelle mesure êtes-vous sûr d'utiliser quelques-unes des compétences que vous avez apprises?" et "Pouvez-vous me donner un exemple de la manière dont vous avez utilisé ou pratiqué une des compétences?"; les attentes de résultat et les croyances relatives à l'importance du coping adéquat grâce à la question "Dans quelle mesure êtes-vous d'accord avec cette phrase Mon mode de coping est important pour mon bien-être"; et enfin, le besoin de discussions additionnelles grâce à la question "Cela vous intéresserait-il d'assister à une séance de suivi?" Les réponses comprenaient une échelle de type Likert en 4 points (allant de "pas du tout" à "beaucoup") qui permettait d'évaluer les intentions, l'autoefficacité et les attentes de résultat ainsi que les croyances des participants.

Puis, nous avons invité les participants à assister à un groupe de réflexion sur les bienfaits de l'intervention et sur les obstacles à son utilité. Dix sujets, huit femmes et deux hommes, ont répondu à l'appel et se sont rencontrés dans le milieu hospitalier où ils ont parlé de leur capacité de coping et du rôle du programme Nucare relativement à leurs habiletés d'adaptation. Les données descriptives émanant du groupe de réflexion ont été examinées au moyen d'une analyse de contenu et d'une recherche de thèmes en regard de l'enquête.

\section{Résultats}

\section{Partie 1: L'enquête}

Vingt-six des 27 participants potentiels étaient des femmes. Sur ces 27 sujets, une femme n'avait pas le temps de participer et une autre ne pouvait participer car elle était hospitalisée pour sa maladie. Quatre femmes et un homme n'ont pu être rejoints, soit un taux de réduction naturelle de $26 \%$. Trois sujets ont répondu partiellement aux questions de l'enquête.

Dix-neuf répondants étaient des patients (95\% de l'échantillon) et le dernier une amie proche d'une patiente (5\% de l'échantillon). Il n'y avait, dans l'échantillon, ni époux ni membres de la famille des patients.

Les indicateurs issus de l'enquête comprenaient les suivants: utilité, intention, auto-efficacité, et enfin, attentes ou croyances. Sept des répondants interrogés (35\%) attribuaient la cote "beaucoup" à l'intention de changer. Trois participants $(15 \%)$ indiquaient qu'ils devaient changer "moyennement", et sept (35\%) rapportaient seulement "un peu de changement".

Huit répondants $(40 \%)$ ont caractérisé leur sentiment d'autoefficacité en se disant "très sûrs" d'utiliser les compétences acquises durant l'intervention. Quatre répondants (20\%) ont dit qu'ils étaient "assez surs" tandis que $30 \%$ avançaient qu'ils étaient "peu sûrs" d'utiliser les compétences. Quatorze répondants (70\%) ont donné un exemple de la manière dont ils utilisaient les compétences apprises. Cela incluait: évaluer la situation avant d'agir, distinguer entre une situation réelle et une perception, faire de la résolution de problème, comprendre le besoin de faire appel à du soutien, et enfin, être capable de rechercher ce soutien. Certains répondants ont signalé qu'ils utilisaient les techniques de relaxation. Une répondante a dit qu'il lui était plus facile d'être franche à propos de ses sentiments et de les communiquer à autrui. Une autre pouvait mieux se valoriser et se sentait moins coupable quand elle disait "non" alors qu'elle avait déjà organisé quelque chose pour ellemême. Une autre répondante a mentionné l'établissement de buts. Certaines ont affirmé qu'elles étaient davantage capables de cerner leurs émotions et leurs pensées.

L'attente de résultat et les croyances étaient évaluées grâce à une question où $95 \%$ des répondants ont indiqué qu'ils avaient de grandes attentes et qu'ils estimaient que le coping est une composante importante du bien-être. Une seule répondante a déclaré avoir des attentes moyennes.
Pour examiner la relation entre ces trois variables, nous avons réalisé une analyse de corrélation de Pearson. Il existait une corrélation positive significative entre l'évaluation du programme et l'autoefficacité $(r=0,48, p<0,05)$ et entre l'évaluation du programme et les attentes et les croyances des participantes $(r=0,45, p<0,05)$. Les autres variables ne comportaient aucune corrélation significative.

\section{Partie 2: le groupe de réflexion}

Dix participants, huit femmes et deux hommes, se sont réunis pour réfléchir à l'intervention Nucare. Une femme a déclaré utiliser le manuel comme encouragement à exécuter les activités normales de la vie. Une autre qui avait été diagnostiquée deux ans plus tôt avait dû faire une demande de prestations d'invalidité après 19 années d'emploi. Son époux était récemment décédé du cancer. Elle se servait du manuel comme guide pour l'aider à faire face à ces stresseurs et à commencer à se donner la permission de faire des choses qui lui plaisaient. Une autre participante a déclaré garder le manuel ouvert sur sa table de chevet et choisir une page au hasard pour la relire. Deux femmes qui avaient pratiqué plus de 30 fois la compétence se rapportant aux façons de penser utilisaient désormais le schéma "faits, pensées et sentiments" afin d'aider leurs enfants à relever les défis auxquels ils devaient faire face dans la vie. Un homme a dit que le matériel présenté lui était connu depuis toujours mais que l'atelier et le manuel lui avaient permis de compartimenter ses connaissances. Dorénavant, il fait un excellent bénévole au sein de L'espoir c'est la vie, l'organisme bénévole de soutien par les pairs qui met en œuvre le programme Nucare.

Une participante a indiqué qu'elle suivait actuellement des cours en modes de vie propices au mieux-être dans une université locale. Une autre participante estimait que lorsqu'elle travaillait, elle débordait d'énergie et se définissait avant tout par son emploi. Désormais, elle a appris, grâce au programme et au manuel, qu'un petit changement fait une différence dans la manière dont elle aborde les grands enjeux de la vie, ce qui lui permet d'améliorer sa qualité de vie. Un participant qui avait été traité pour un lymphome non hodgkinien cinq ans plus tôt exprimait le besoin de rester actif. Il est bénévole à l'hôpital et se consacre à la peinture et à d'autres activités. Il a ajouté qu'il passait toujours une excellente journée à donner et à recevoir du soutien social. Une femme récemment diagnostiquée avait pu recadrer sa maladie pour en faire sa "petite montagne", plutôt qu'une force dominant complètement sa vie. Elle apprenait à y faire face en établissant de petits buts.

Un des hommes jugeait que l'intervention l'aidait à acquérir des compétences pour changer sa façon de penser et à structurer sa pensée. Il pouvait ainsi accorder à la maladie l'importance qui lui revenait, sans plus, et donc d'avoir assez de place pour les autres aspects de sa vie et l'établissement de buts. Une patiente atteinte d'un cancer de stade avancé a signalé que le programme l'aidait à structurer sa vie. En étudiant l'outil "faits, pensées et sentiments", elle se sentait davantage capable de faire face à sa maladie et à d'autres événements de sa vie. Une autre patiente a indiqué qu'elle s'encourageait tout au long de son traitement en disant tout haut, chaque jour, qu'elle allait gagner la lutte. Cependant, elle sentait que son but de "victoire éventuelle" était très ardu et qu'il représentait un objectif difficile à atteindre. Son amie, qui avait également participé à l'atelier, lui a acheté une casquette des Jeux olympiques dont elles ont fait le symbole des hauts et des bas de son traitement anticancéreux qui leur rappelait en même temps le but ultérieur. Elle a ajouté qu'elle s'était sentie soutenue lorsque son amie lui avait offert la casquette et qu'elle a réalisé alors que son amie comprenait ses inquiétudes et ses peurs. Son amie a déclaré qu'elle était plus capable d'offrir un soutien réel et de faire partie de l'équipe.

Les thèmes issus du groupe de réflexion semblaient refléter l'autoefficacité et l'apprentissage qui se produisaient au cours des séances. Les exemples donnés ci-dessus révèlent l'utilisation des compétences et des résultats bénéfiques d'une grande importance pour les participants. 
D'après les participants, le manuel constituait une partie importante de l'intervention. La plupart d'entre eux disaient qu'ils le consultaient fréquemment, qu'ils y trouvaient la confirmation de ce qu'ils essayaient d'accomplir; ils éprouvaient un véritable sentiment de sécurité à l'idée de savoir qu'il demeurait à portée de la main.

Deux aspects de Nucare semblaient revêtir une importance particulière aux yeux des participants: 1) Cela les rassurait de savoir qu'il suffisait d'une petite dose de changement pour obtenir des résultats positifs. Les participants s'estimaient capables de faire de petits changements plutôt que les changements de grande envergure qu'ils supposaient nécessaires. 2) Les participants ont pratiqué certaines des compétences, après avoir saisi qu'une telle pratique est essentielle si on veut que ces compétences deviennent une seconde nature.

Les participants ont exprimé l'importance de l'intervention à la lumière de l'augmentation de leur auto-efficacité. Ils ont appris à se donner la permission de faire ce qu'ils voulaient, y compris de passer du temps à se faire plaisir sans se sentir coupable. Le fait d'établir des petits buts atteignables les a également aidés à accroître leur confiance.

\section{Discussion}

Le manuel représentait une partie importante de l'intervention. De nombreux participants ont signalé avoir eu besoin de consulter fréquemment le matériel, mais aussi d'avoir voulu le faire. Certains sujets ont utilisé le manuel comme guide en vue d'apprendre les habiletés d'adaptation et de renforcer celles qu'ils maîtrisaient déjà. Le manuel leur permettait de valider leurs sentiments et de diriger leur attention sur eux-mêmes sans pour autant éprouver de culpabilité. Il est évident que le matériel écrit joue un rôle important en matière de renforcement, de soutien et de connaissances.

De nombreuses études, y compris la nôtre, n'incorporaient pas de données reflétant l'ampleur des pratiques effectuées au domicile (Devine et Westlake, 1995). Il nous est donc impossible d'appuyer ou au contraire de mettre en doute l'hypothèse voulant que la pratique ait une incidence sur les résultats. Toutefois, on constate qu'il était important pour les participants de savoir que la pratique est un élément intrinsèque du changement et que les changements peuvent être de petite envergure.

Bandura (1977) a avancé que les individus sont exposés, tout au long de leur vie, à diverses expériences de modification du comportement et de l'auto-efficacité. Ainsi, le simple ajout d'une intervention ne touchera pas tout le monde de manière uniforme. La gamme des réponses relatives à l'utilité de chacune des compétences enseignées dans le cadre de l'intervention allait de "pas du tout" à "beaucoup". Bien que l'enquête n'ait pas examiné les expériences passées, nous avons supposé que certains participants avaient déjà été exposés à d'autres situations propices à l'amélioration des habiletés d'adaptation. Cette théorie est également avalisée par la gamme des réponses en matière de perception de l'auto-efficacité, qui allait de "un peu" à "beaucoup" où les sujets qui avaient des compétences supérieures dès le départ se sentaient plus confiants que les autres.

Quoique $35 \%$ des participants aient dit qu'il leur suffisait de faire un petit changement dans leur coping, il est intéressant de noter leur pleine participation au programme. Il se peut que les patients cherchent à valider leurs stratégies de coping actuelles et qu'ils tirent donc des avantages d'un tel programme. Leur intention d'explorer de nouvelles manières de coping ou de réaffirmer leur pratique actuelle semblait encourager leur participation à l'intervention. Les répondants ont retenu la gamme allant de "un peu" à "beaucoup" pour définir leur besoin de changement, appuyant par là même leur décision de participer à l'intervention. L'intention de changer est un précurseur de la tentative de changement mais elle ne se traduit pas nécessairement par le désir ou la capacité de changer au cours de la même période ou même du tout (Whitehead, 2001).

Prochaska et Velicer (1997) ont décrit les cinq étapes relatives à la prise de décision qui sont essentielles à la mise en œuvre du changement: la précontemplation où l'individu ne pense pas au changement; la contemplation où l'individu pense qu'il a besoin de faire des changements, réfléchit aux choix qu'il doit faire et examine les options; la préparation où l'individu commence à prendre des mesures de petite envergure pour changer; l'action où les changements que l'individu a mis en œuvre sont encore de courte durée, soit moins de six mois; et enfin, l'entretien où les changements effectués par l'individu sont de longue durée, soit plus de six mois. Il semble que les participants au programme Nucare en sont à l'étape de la contemplation - où ils rassemblent de l'information - ou à l'étape de la préparation où ils commencent à prendre des mesures de petite envergure en vue de changer. On pourrait demander aux patients de se pencher sur l'étape de capacité de changement à laquelle ils se trouvent.

La corrélation modérée mais significative entre le haut degré d'utilité accordée à l'intervention et l'auto-efficacité nous amène à penser que le désir d'améliorer leur auto-efficacité faisait partie intégrante de leur décision d'assister aux séances. Cela concerne la capacité d'apprentissage des participants et le moment de leur participation à l'intervention tel que choisi par eux-mêmes.

Deux concepts, soit qu'il suffit d'une petite dose de changement et qu'il faut pratiquer une nouvelle compétence de 30 à 60 fois pour qu'elle devienne une seconde nature, sont proposés et continuellement renforcés dans le manuel et dans les séances Nucare.

Les répondants ont exprimé des attentes positives en matière de résultats de développement d'habiletés de coping. Ils croyaient entre "assez" et "beaucoup" que le coping constitue un élément important du bien-être. Cette croyance sous-tend la décision des sujets de participer à l'atelier dans l'espoir d'améliorer leur capacité à faire face aux épreuves. C'est peut-être parce qu'ils croyaient que le coping est important que les participants ont assisté aux séances ou alors, il se peut que l'atelier ait renforcé cette croyance. La corrélation n'indique pas la causalité, mais qu'il existe bel et bien un lien.

\section{Limites}

Ces résultats ne peuvent pas être généralisés du fait de la petite taille de l'échantillon qui, de plus, est non aléatoire. L'impact des résultats serait rehaussé par l'élargissement du programme Nucare à d'autres centres de soins, l'inclusion de questions sur l'auto-efficacité et sur les étapes de la capacité à changer ainsi que par le suivi à long terme des participants.

\section{Répercussions pour les soins infirmiers}

Cette étude constitue un examen important de cette intervention et des conséquences qu'elle a pour les soins infirmiers et de notre entendement de la manière dont les patients font des choix en matière de coping. Quand elles ont conscience de l'importance que les participants accordent au coping en matière de bien-être, les infirmières sont capables de planifier des interventions propices à l'adaptation pour les patients et leurs proches. En tenant compte du fait que l'intention de changer et la capacité de changement ne vont pas nécessairement de pair, les infirmières peuvent favoriser la promotion du changement et faciliter les décisions amenant au changement en utilisant la famille comme "unité de traitement", en soulignant à tous ses membres que l'intention de changer, à elle seule, est un premier pas important.

Puisque les résultats montrent que l'intervention Nucare est liée à une auto-efficacité croissante, les infirmières devraient examiner la possibilité d'enseigner des habiletés de coping aux patients et à leurs proches et de les promouvoir, en utilisant Nucare comme guide de pratique. Un aspect de l'intervention que les participants ont trouvé particulièrement utile était les fréquents rappels sur la nécessité des pratiques et sur le fait que même une petite dose de changement peut s'accompagner de résultats. Il serait utile d'organiser des séances de suivi à divers intervalles étant donné que $65 \%$ des répondants ont indiqué qu'ils aimeraient avoir accès à ce type de séances. 


\section{Recherches futures}

Au cours des douze derniers mois, plus de 150 patients et amis ou proches de patients ont participé au programme Nucare. Nous offrons le programme dans la journée et dans la soirée afin de répondre aux besoins de la population que nous desservons. Les recherches futures pourraient essayer de cerner des solutions aux obstacles à la participation dégagés par le groupe de réflexion tels que le transport, les responsabilités familiales et les horaires de travail.

Les recherches pourraient également porter sur la participation à des programmes similaires. Elles pourraient examiner les stades où se situent les participants en matière de changement et de prise de décision ainsi que leur capacité d'apprentissage. Cela pourrait avoir un effet sur le moment choisi pour chacune des interventions et sur ce qu'on devrait offrir aux sujets afin de maximiser leur capacité d'adaptation.

\section{Conclusion}

L'étude indique qu'il existe des corrélations entre le haut degré d'utilité accordé à l'intervention et l'augmentation de l'auto-efficacité et de la croyance que le coping constitue un élément important du bien-être. Le choix du moment peut être une composante importante de la réussite de l'apprentissage; ainsi, on laisse aux participants le soin de choisir quand ils souhaitent assister aux séances en leur donnant par là même l'occasion de décider quand ils se sentent prêts à le faire Bien que les patients soient prêts à se renseigner davantage sur les habiletés de coping, cela ne veut pas dire qu'ils sont prêts à changer ou capables de le faire. Par contre, ils ont acquis de nouvelles connaissances et armés de leur manuel, ils peuvent réviser l'information dispensée et pratiquer les compétences lorsqu'ils se sentent prêts. Deux aspects de l'atelier sont particulièrement dignes d'attention et peuvent être incorporés dans les soins infirmiers aux patients atteints de cancer: il faut pratiquer les nouvelles habiletés de coping et une petite dose de changement suffit pour obtenir des résultats. De courts ateliers psychopédagogiques sur le coping avec des séances de suivi représentent sont une nouvelle technique utile pour les soins aux patients atteints de cancer et ils appartiennent au champ de la pratique des soins infirmiers en oncologie.

\section{Références}

Anderson, E.S., Winett, R.A., \& Wojcik, J.R. (2000). Social-cognitive determinants of nutritional behaviour among supermarket food shoppers: A structural equation analysis. Health Psychology, 19(5), 479-486

Babcock, D.E., \& Miller, M.A. (1994). Client education: Theory and practice. St. Louis: Mosby.

Bandura, A. (1977). Self-efficacy: Toward a unifying theory of behavioral change. Psychological Review, 84(2), 191-215.

Bandura, A. (1997). Self-efficacy: The exercise of control. New York: Freeman

Devine, E.C., \& Westlake, S.K. (1995). The effects of psychoeducational care provided to adults with cancer: Meta-analysis of 116 studies. Oncology Nursing Forum, 22(9), 1369-1381.

Edgar, L., Remmer, J., Rosberger, Z., \& Fournier, M. (2000). Resource use in women completing treatment for breast cancer. Journal of Psychosocial Oncology, 9(5) 428-438.

Edgar, L., Rosberger, Z., \& Collet, J.C. (2001). Lessons learned: Outcomes and methodology of a coping skills intervention trial comparing individual and group formats for patients with cancer. International Journal Psychiatry in Medicine, 31(3), 3-5-320.

Edgar, L., Rosberger, Z., Collet, J.C., \& Fournier, M. (2002). Patterns of coping in women completing treatment for breast cancer. Journal of Psychosocial Oncology, 20(3), 19-37.

Edgar, L., Rosberger, Z., \& Nowlis, D. (1992). Coping with cancer during the first year after diagnosis. Cancer, 69(3), 817-828.

Farber, J.M., Weinerman, B.H., \& Kuypers, J.A. (1984). Psychosocial distress in oncology outpatients. Journal of Psychosocial Oncology, 2(3-4), 109-118.

Folkman, S., \& Lazarus, R.S. (1988). The relationship between coping and emotion: Implications for theory and research. Journal of Social Science and Medicine, 26(3), 309-317.

Gottlieb, L.N., \& Rowat, K. (1987). The McGill model of nursing: A practice-derived model. In L.N. Gottlieb \& H.E. Ezer (Eds.), Health, family, learning \& collaborative nursing (pp. 253-263). Montreal: McGill University School of Nursing.

Grahn, E.B.M., Stigmar, G.K.E., \& Ekdahl, C.S. (2001). Motivation for change and personal resources in patients with prolonged musculoskeletal disorders. Journal of Bodywork and Movement Therapies, 5(3), 160-172.
Hardy, G.E., Barkham, M., Shapiro, D.A., Reynolds, S., \& Rees, A. (1995). Credibility and outcome of cognitive-behavioral and psychodynamic-interpersonal psychotherapy. British Journal of Clinical Psychology, 34, 555-569.

Jackson, K.M., \& Aiken, L.S. (2000). A psychosocial model of sun protection and sunbathing in young women: The impact of health beliefs, attitudes, norms and self-efficacy for sun protection. Health Psychology, 19(5), 469-478.

Lipshitz, R., \& Strauss, O. (1997). Coping with uncertainty: A naturalistic decision-making analysis. Organizational Behavior and Human Decision Processes, 69(2), 149-163.

Prochaska, J.O., \& Velicer, W.F. (1997). The transtheoretical model of health behavior change. American Journal of Health Promotion, 12(1), 38-48.

Santé Canada. (1997). Principales causes de décès et d'hospitalisation au Canada. Récupéré le 18 août 2003, à l'adresse: http://www.hc-sc.gc.ca/pphb-dgspsp/publicat/lcdpcd97/mrt_mf_f.html

Schwarzer, R., \& Renner, B. (2000). Social-cognitive predictors of health behaviors: Action Self-efficacy and coping self-efficacy. Health Psychology, 19(5), 487-495.

Senécal, C., Nouwen, A., \& White, D. (2000). Motivation and dietary self-care in adults with diabetes: Are self-efficacy and autonomous self-regulation complementary or competing constructs? Health Psychology, 19(5) 452-457.

Statistiques canadiennes sur le cancer. (2003). Récupéré le 18 août 2003, à l'adresse: http:www.cancer.ca

Trask, P.C., Paterson, A.G., Hayasaka, S., Dunn, R.L., Riba, M., \& Johnson, T. (2001). Psychosocial characteristics of individuals with non-stage IV melanoma. Journal of Clinical Oncology, 19(11), 2844-2850.

Trask, P.C., Schwartz, S.M., Deaner, S.L., Paterson, A.G., Johnson,T., Rubenfire, M., \& Pomerleau, O.F. (2002). Behavioral medicine: The challenge of integrating psychological and behavioral approaches into primary care. [Review]. Effective Clinical Practice, 5(2), 75-83.

Whitehead, D. (2001). A social cognitive model for health education/health promotion practice. Journal of Advanced Nursing, 36(3), 417-425. 